\title{
Improved categorization of subtle facial expressions modulates Late Positive Potential
} (LPP).

Petra M. J. Pollux (PhD), School of Psychology University of Lincoln LN6 7TS, Lincoln, UK. E-mail: ppollux @lincoln.ac.uk, Tel: +44(0)1522886360

\begin{abstract}
Biases in facial expression recognition can be reduced successfully using feedback-based training tasks. Here we investigate with Event-Related Potentials (ERP) at which stages of stimulus processing emotion-related modulations are influenced by training. Categorization of subtle facial expressions (morphed from neutral to happy, sad or surprise) was trained with correct-response feedback on each trial. ERPs were recorded before and after training whilst participants categorized facial expressions without response feedback. Behavioural data demonstrated large improvements in categorization of subtle facial expression which transferred to new face models not used during training. ERPs were modulated by training from $450 \mathrm{~ms}$ post-stimulus onwards, characterized by a more gradual increase in P3b/LPP amplitude as expression intensity increased. This effect was indistinguishable for faces used for training and for new faces. It was proposed that training elicited a more fine-grained analysis of facial information for all subtle expressions, resulting in improved recognition and enhanced emotional motivational salience (reflected in P3b/LPP amplitude) of faces previously categorized as expressing no emotion.
\end{abstract}

Key-words: Facial expression categorization, training, ERP. 


\section{INTRODUCTION}

The ability to make rapid judgements of facial expressions in order to interpret social situations and to flexibly adjust behaviour to the social environment is essential for successful social interaction. Expression recognition skills vary in the general population (Sullivan et al, 2007; Vassalo et al, 2009; Werheid et al, 2010; Tamamiy and Hiraki, 2013) and have been associated with social adjustment, mental health and workplace performance (Carton et al, 1999; Norwicky and Duke, 1994). Specific impairments in facial expression categorization have been associated with a range of neurological and psychological problems, such as amygdala lesions (Adolphs et al, 2005), autism (Baron-Cohen, 1995), schizophrenia (Edwards et al, 2002; Penn et al, 2006), social anxiety (Heuer et al, 2010) and major depression (Joormann and Gotlieb, 2006). As a result, different methodologies aimed at improving categorization of facial expressions have been developed over time. Some approaches have been targeted at specific populations, such as people with autism (Golan et al, 2010), amygdala lesions (Adolphs et al, 2005) and schizophrenia (Combs et al, 2011; Sachs et al, 2012; Luckhaus et al, 2013) whereas other training tasks may be useful for a wider population. One such methodology is aimed at modifying perceptual biases in categorization of ambiguous facial expression. Perceptual biases in facial expression categorization have been associated with exposure and social experiences (e.g., Pollak et al, 2009) and have been observed in people with major depression (Joormann and Gottlieb, 2006), in abused children (Pollak et al, 2009; Da Silva Ferreira et al, 2014) and in adolescents at high risk of aggression and delinquency (Penton-Voak et al, 2013).

A few studies have shown that feedback-based training can be used to reduce categorization biases for subtle facial expressions (Penton-Voak et al, 2013; Pollux et al, 2014). Penton-Vaok et al (2013) showed for example that ambiguous facial expressions 
(created by morphing angry and happy faces), perceived as angry before feed-back training, were more likely categorized as happy after having been relabelled as happy during training in young adolescence at high risk of aggression and delinquency. Similarly, categorization accuracy of subtle facial expressions (created by morphing neutral with highly intense facial expressions) improves in typically developing children after feedback-based training where subtle facial expressions previously perceived as neutral were relabelled with the morphed expression during training (Pollux et al, 2014). Moreover, these training effects are not stimulus specific: Changes in expression recognition responses seem to transfer to new face models after training (Penton-Voak et al, 2013; Pollux et al, 2014) and even to subjective experience of emotional state, such as lower levels of state anger, after successful reduction of anger-bias (Penton-Voak et al, 2013).

Despite the growing interest in this type of feedback-based training methodologies, the neural processes underlying training-related changes in facial expression categorization are not yet fully known. The event-related potential (ERP) methodology measures changes in event-related electrical activity from the scalp with a high temporal resolution and thus allows analysis of signal modulation at different stages of stimulus processing. The aim of the current study is to investigate training-related changes in ERP measures that are known to be modulated by facial expression, using a feedback-based training task which is aimed at improving expression categorization responses for subtle facial expressions.

ERP components that are often reported to be enhanced by facial expression compared to neutral faces are P1 (Batty and Taylor, 2003; Luo et al, 2010; Zhang et al, 2013), N170 (Williams et al, 2006; Sprengelmeyer and Jentzsch, 2006; Utama et al, 2009; Luo et al, 2010; Zhang et al, 2013) and P3b/LPP (Eimer and Holmes, 2002; Luo et al, 2010; Duval et al, 2013). P1 modulation tends to be most pronounced for fearful faces and has been suggested to reflect automatic rapid detection of biological salient or threatening information 
(Pourtois et al, 2004; Vuileumier and Pourtois, 2007). N170, suggested to be face-specific (Benton and Deouell, 2000; Itier and Taylor, 2004; Sadeh et al, 2010) and to be associated with structural encoding of faces (Rossion et al, 1999), has been shown to be modulated by emotional faces (Williams et al, 2006; Sprengelmeyer and Jentzsch, 2006; Utama et al, 2009; Luo et al, 2010; Zhang et al, 2013, Hinojosa et al, 2015; but see Eimer and Holmes, 2002; Eimer et al, 2003) and its amplitude has been found to enhance gradually as intensity of facial expressions increases (Sprengelmeyer and Jentzsch, 2006; Utama et al, 2009). This emotion sensitivity has been suggested to reflect an early stage in facial expression processing where sufficient information is available to distinguish emotional from neutral faces (Luo et al, 2010; Zhang et al, 2013). The long-latency potentials P3b and the Late Positive Potential (LPP) have been related to post-perceptual stages, where incoming information is processed further to enable decisions about the specific nature of the emotional content (Cuthbert et al, 2000; Schupp et al, 2000; Campanella et al, 2002; Oloffson et al, 2008; Hajcek and MacNamara, 2010). Enhancement of P3b/LPP amplitude by photographs of positive or negative scenes compared to neutral images has been associated with greater allocation of attentional processing resources due to the motivational relevance of the emotional content (Cuthbert et al, 2000; Schupp et al, 2000; Oloffson et al, 2008; Hajcek and MacNamara, 2010). A similar modulation of P3b/LPP has been found for emotional compared to neutral faces (Eimer and Holmes, 2002; Luo et al, 2010; Duval et al, 2013). Moreover, P3b/LPP amplitude enhances gradually as the intensity of facial expressions increases (Duval et al, 2013), supporting the idea that emotion in faces enhances their motivational salience and increases allocation of attentional resources for further processing (Oloffson et al, 2008; Hajcek and MacNamara, 2010; Duval et al, 2013).

To investigate modulations of ERP by improved categorization of subtle facial expressions in the present study, face images were morphed from neutral to happy, sad and 
surprise and were presented in a feedback-based facial expression categorization training task. ERPs were recorded before and after training using a similar task without response feedback, and transfer of learning was measured by including a face-set that was not used for training. The choice of task (adapted from Pollux et al, 2014) was based on the reported effect of facial expression intensity on N170 (Sprengelmeyer et al, 2006; Utama et al, 2009) and P3b/LPP (Duval et al, 2013) which allows generation of specific predictions for trainingrelated amplitude modulations. Consistent with the observation that N170 enhances as expression intensity increases, previously associated with discrimination between emotional and neutral expressions (Luo et al, 2010; Zhang et al, 2013), amplitude should enhance for low-intensity expressions that are more likely perceived as neutral before training and more often categorized as emotional afterwards. Moreover, these re-categorized faces should attract more attentional resources after training as their motivational salience increases and should therefore be associated with enhanced P3b/LPP amplitude (Duval et al, 2013). As a result, training could result in a more gradual increase of N170 and P3b/LPP elicited by low intensity facial expressions after training compared to before. In addition, based on previous studies showing higher accuracy for low intensity happy compared to subtle sad and surprised (Guo, 2012; Pollux et al, 2014), the N170 and P3b/LPP modulation may be more pronounced for happy faces.

\section{METHODS}

\subsection{Participants}

Sixteen participants (13 women and 3 men, mean age is $20.3 \pm 0.6$ years) were recruited from the Psychology Subject Pool for course credit and a voucher worth $£ 20$. All participants had normal or to-normal-corrected vision. Ethical approval was obtained from 
the Ethics Committee in the School of Psychology, University of Lincoln. All procedures complied with the British Psychological Society "Code of Ethics and Conduct" and with the World Medical Association Helsinki Declaration as revised in October 2008.

\subsection{Materials and Stimuli}

Digitised grey-scale face images in full frontal view were displayed on a highfrequency non-interlaced gamma-corrected colour monitor $(100-\mathrm{Hz}$ frame rate, $1024 \times 768$ pixels, Mitsubishi Diamond Pro 2070SB) with a uniform grey background $(24 \mathrm{~cd} / \mathrm{m} 2$ luminance). At a viewing distance of $57 \mathrm{~cm}$, the monitor subtended a visual angle of $40 \times 30$ deg. Four western Caucasian faces (two female and two male models) were selected from the Karolinska Directed Emotional Faces (Lundqvist et al, 1998). Each model expressed no emotion (neutral), happiness, sadness, or surprise at high intensity. The choice of expressions was partly based on the reliable training-effects observed previously for sad and happy expressions (Pollux et al, 2014). Surprise was chosen to balance positive with negative expressions (surprise could be interpreted as positive or negative) and to include an expression that is generally associated with lower accuracy, particularly for lower intensity levels (Guo et al, 2012). The faces were processed in Adobe Photoshop to remove external facial features and to ensure a homogenous grey background, same face size and brightness. A black oval cut-out shape covered the hair to ensure that only facial features were visible. Morpheus Photo Morpher was used to create expression intensity levels ranging from 5 to $100 \%$ with $5 \%$ increments by morphing the emotional face with the neutral face.

To find out at which intensity levels training would be maximal, 8 participants ( 6 women and 2 men, mean age $24.6 \pm 0.8$ years) were trained in a pilot study consisting of three sessions. Expression intensity levels included were $0 \%$ (neutral), 10\%, 15\%, 20\%, 25\%, 30\%, $35 \%, 40 \%, 50 \%$ and $100 \%$, for each emotion. Each trial commenced with a fixation in the 
centre of the screen $(500 \mathrm{~ms})$ which was replaced by an emotional face for $250 \mathrm{~ms}$.

Participants were instructed to identify the facial expression by pressing one of four response keys, where each key corresponds to one of four emotions (neutral, happy, sad or surprised). Participants were warned that expressions could be very subtle. Feedback for each response was either 'yes, correct', or 'no, incorrect', the latter being followed by the correct response. Responses were made with the preferred hand on a purpose built button box, where the position of the keys was adjusted to the natural position of the fingers when the hand is resting on a flat surface. The button box sloped slightly upward from the body to the top of the button box where the response keys were located to ensure a comfortable position and to reduce noise due to muscle movements during the EEG sessions. Two faces with 10 intensity levels of three emotions were repeated 10 times in each session (600 trials per session). Duration of each testing session was approximately 40 minutes. The results of the pilot showed that performance increased most for the lower intensity expressions. Percentage improvement from the first block of 200 trials in session 1 to the last 200 trials in session 3 was $29.9,32.2,22.6,9.7,4.2,1.3,1.3,0.8$ and $1.2 \%$ for intensity levels $10,15,20,25,30,35$, 40, 50 and $100 \%$, respectively. Based on these pilot data, only the lowest intensity levels (10, 15 and $20 \%$, in addition to $0 \%$ and $100 \%$ ), were included for the task used for recording ERP before and after the three training sessions in the main experiment.

The five sessions of the main experiment (ERP-session, 3 training sessions, ERPsession) took place on 5 successive days in a soundproof and electrically shielded room. The faces of all four models were presented in the pre-and post-test sessions whereas only 2 models were used for training, allowing investigation of transfer-effects of training to new faces. The face-set used for training was counterbalanced across participant. The task used for the three training sessions in the main experiment was identical to the task used in the pilot study, where feedback was provided on all trials (see above for details). The event 
sequence for the pre-and post-test was as follows: Each trial started with a fixation at the centre of the screen for $500 \mathrm{~ms}$, followed by an image of an emotional face for $250 \mathrm{~ms}$. The face was replaced by the fixation point for $1000 \mathrm{~ms}$ or until a response was made. At that point, the fixation point disappeared for one second before reappearing again for the next trial. Participants were encouraged to maintain fixation on the fixation point and to use the $1000 \mathrm{~ms}$ without fixation to blink if necessary. For the ERP sessions, there were 25 repetitions for each emotional face at each intensity level and 75 for the neutral expression for each model, resulting in 1500 trials. The trials were divided into five blocks of 300 trials, each consisting of 60 neutral faces ( 4 models $\times 15$ repetitions) and 240 emotional faces ( 4 models $\times$ 5 repetitions $\times 4$ intensity levels). The task was paused after every 50 trials for a duration determined by the participant. Pre-and post-test sessions (including preparation of participants for EEG recording) lasted approximately 2.5 hours.

\subsection{ERP recording and analysis}

EEG was recorded with a sampling rate of $1048 \mathrm{~Hz}$ from 64 scalp locations using active Ag-AgCL-tipped electrodes attached to an electrode cap using the 10/20 labelling systems. The Active Two system (Biosemi, Amsterdam) was used for recording. EEG signals were referenced during the recording to an additional active electrode (Common Mode Sense). In addition to the electrode cap, four electrodes were used to record electrooculograms (EOGs). Two electrodes were placed at the outer canthi of both eyes (horizontal EOG) and two electrodes were placed on infraorbital and supraorbital locations of the right eye (vertical EOG). Two additional electrodes (placed behind the left and right ear) were used for the off-line linked-mastoid referencing process. Signals were filtered off-line (highpass filter: $0.16 \mathrm{~Hz}$; low-pass filter: $70 \mathrm{~Hz}$ ). Segments were time-locked to $300 \mathrm{~ms}$ before the onset of stimulus presentation. Raw EEG was first segmented in epochs of 2300ms. Trials 
with horizontal eye-movements were removed, either after detected by visual inspection or by automatic rejection of segments with amplitude change $>180 \mu \mathrm{V}$ or with amplitudes exceeding the amplitude criterion $( \pm 100 \mu \mathrm{V})$ within $200 \mathrm{~ms}$ intervals at the scalp electrodes. After visual inspection, mean amplitudes were extracted from O1, O2, P3, P4, P5, P6, PO3, PO4 (for P1), P7, P8, TP7, TP8 (for N170), CP3, CP1, CPz, CP2, CP4 (for LPP), to establish at which electrodes the components of interest were most pronounced or differentiated most between the different intensity levels. The electrodes and time-windows selected for the final analysis of mean amplitude and peak-latency were PO3/PO4 (90-130) for P1, P7/P8 (130200) for N170 and CP1/CP2 for P3b/LPP (350-1550ms in 100ms time-windows).

\section{RESULTS}

\subsection{Behavioural measures: Pre- and Post-test}

Accuracy: Percentage correct responses were entered in ANOVA with the factors Training (pre-and post- training session), Face-set (training set vs. 'new' face set in the posttraining session), Block (blocks 1-5, 300 trials per Block), Expression (happy, sad or surprise) and Intensity (I10, I15, I20, I100). Neutral expressions were analysed separately with the factors Training, Face-set and Block. Greenhouse-Geisser correction was applied where appropriate and Bonferroni t-tests correction was used for post-hoc pair-wise comparisons. Only significant effects are reported.

Figure 1 illustrates the strong beneficial effect of training on accuracy for both face-sets. Analysis of percentage correct responses revealed significant effects of Training $\left[\mathrm{F}(1,15)=104.9 ; \mathrm{p}<0.001, \eta_{p}{ }^{2}=0.87\right]$, Expression $\left[\mathrm{F}(2,30)=14.3 ; \mathrm{p}<0.001, \eta_{p}{ }^{2}=0.62\right]$ and Intensity $\left[\mathrm{F}(3,45)=255.7 ; \mathrm{p}<0.001, \eta_{p}{ }^{2}=0.94\right]$. Accuracy was highest for happy and sad compared to surprised expressions (all $p$ 's $<0.001$ ), and performance increased gradually with 
intensity (all differences between intensity levels were significant $\left(p^{\prime} s^{<}<0.001\right)$ ). The effect of Block was different before and after training $\left[\mathrm{F}(4,60)=2.09 ; \mathrm{p}=0.039, \eta_{p}{ }^{2}=0.15\right]$ : Accuracy was lower in block 1 at pre-test compared to the 4 subsequent blocks (all $p$ 's<0.05), whereas this effect was absent at post-test. Significant effects were further found for Face-set $\left[\mathrm{F}(1,15)=9.4 ; \mathrm{p}=0.008, \eta_{p}{ }^{2}=0.38\right]$, Face-set $\times$ Expression $\left[\mathrm{F}(2,30)=6.9 ; \mathrm{p}=0.003, \eta_{p}{ }^{2}=0.31\right]$, Face-set $\times$ Intensity $\left[\mathrm{F}(3,45)=7.42 ; \mathrm{p}<0.001, \eta_{p}{ }^{2}=0.33\right]$, Training $\times$ Face-set $\times$ Intensity $\left[\mathrm{F}(3,45)=6.24 ; \mathrm{p}<0.001, \eta_{p}{ }^{2}=0.29\right]$, Training $\times$ Expression $\times$ Intensity $[\mathrm{F}(6,90)=3.77$; $\left.\mathrm{p}=0.002, \eta_{p}{ }^{2}=21\right]$ and Training $\times$ Face-set $\times$ Expression $\times$ Intensity $[\mathrm{F}(6,90)=2.8 ; \mathrm{p}=0.015$, $\left.\eta_{p}{ }^{2}=0.18\right]$. Post-hoc analysis of the four-way interaction effect revealed that these interaction effects could all be explained by an isolated effect of face-set after training: A significant difference between trained and new faces was only found in the post-test for the expression surprise at the three lower intensity levels (for all three intensity levels, $p$ ' $s \leq 0.015$ ). Analysis of the neutral condition only revealed a significant effect of Training $[F(1,15)=46.4 ; p<0.001$, $\left.\eta_{p}{ }^{2}=0.76\right]$ due to a reduction in accuracy after training (from $61 \pm 5.7 \%$ to $46.5 \pm 4.95 \%$ ). 

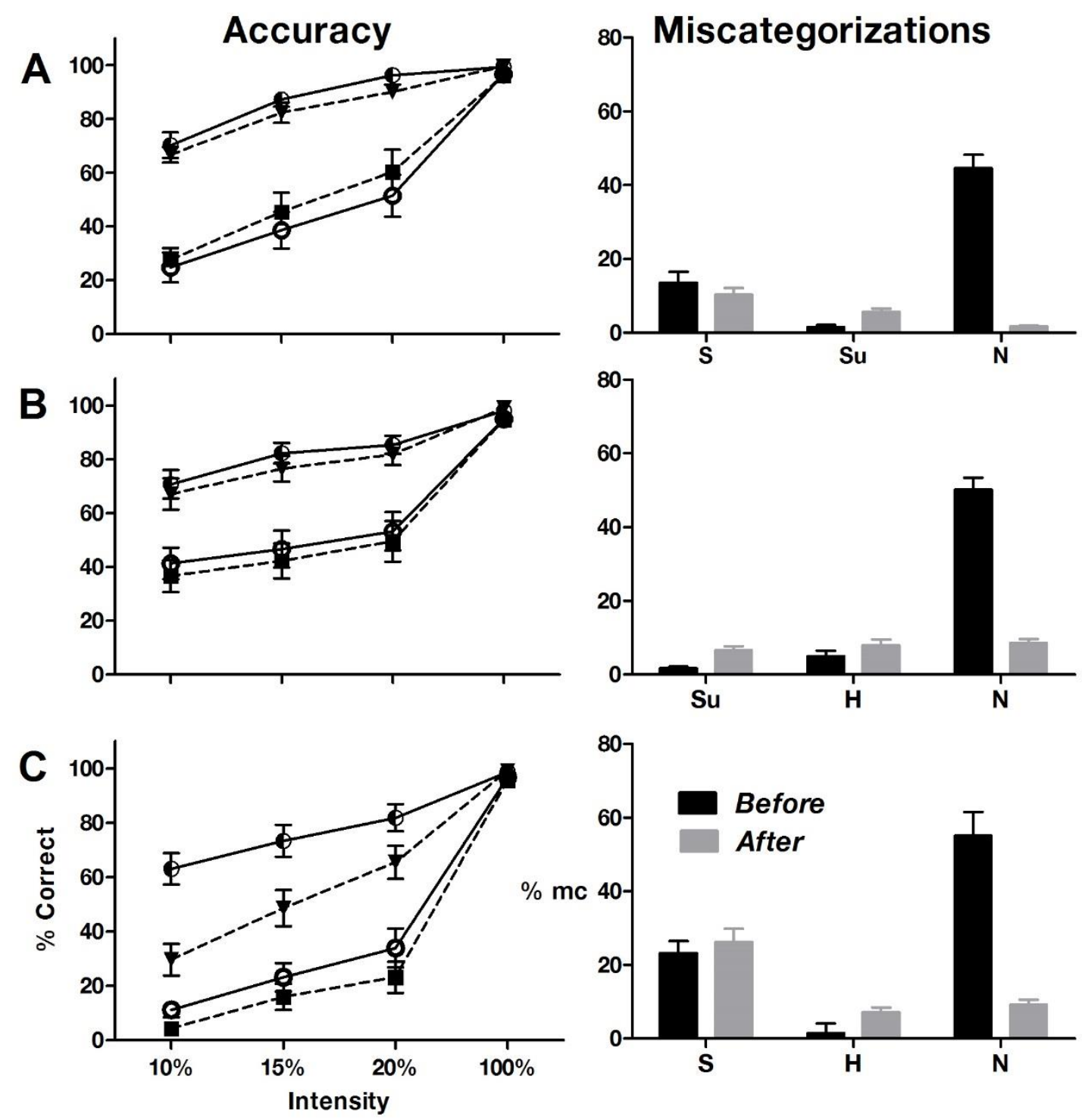

\section{Before ' $T$ ' faces \\ Before ' $N$ ' faces}

Figure 1: Left column: Percentage correct responses (\% correct) for faces-set used for training ('T-faces') and faces not used for training ('Nfaces') by Intensity and Expression ( $A=$ Happy, $B=S a d, C=$ Surprised). Right column: Percentage miscategorizations for low expression intensity only $(10 \%, 15 \%$ and $20 \%)$, collapsed over intensity level and face-set, before and after training (H=Happy, $S=S a d, S u=$ Surprised, $N=$ Neutral. 
Response times: In analysis of mean response times (RT, see Figure 3), significant effects were found for Expression $\left[\mathrm{F}(2,30)=18.1 ; \mathrm{p}<0.01, \eta_{p}{ }^{2}=0.57\right]$, Intensity $[\mathrm{F}(3,45)=31.5$; $\left.\mathrm{p}<0.001, \eta_{p}{ }^{2}=0.69\right]$, Training $\times$ Expression $\left[\mathrm{F}(2,30)=5.8 ; \mathrm{p}=0.008, \eta_{p}{ }^{2}=0.29\right]$ and Training $\times$ Intensity $\left[\mathrm{F}(3,45)=13.6 ; \mathrm{p}<0.001, \eta_{p}{ }^{2}=0.49\right]$. At post-test only, responses were significantly faster to happy expressions than to sad or surprised faces ( $p$ 's $\leq 0.03)$. Only after training, RT decreased significantly with each increment of intensity (for all comparisons, $p$ 's $<0.001$ ), resulting in a significant difference in $\mathrm{RT}$ before and after training for faces with the highest intensity level $(\mathrm{p}=0.02)$. Analysis of the neutral condition revealed a significant effect of Training $\left[\mathrm{F}(1,15)=4.89 ; \mathrm{p}=0.044, \eta_{p}{ }^{2}=0.26\right]$ : Responses times were longer after training on neutral expressions.

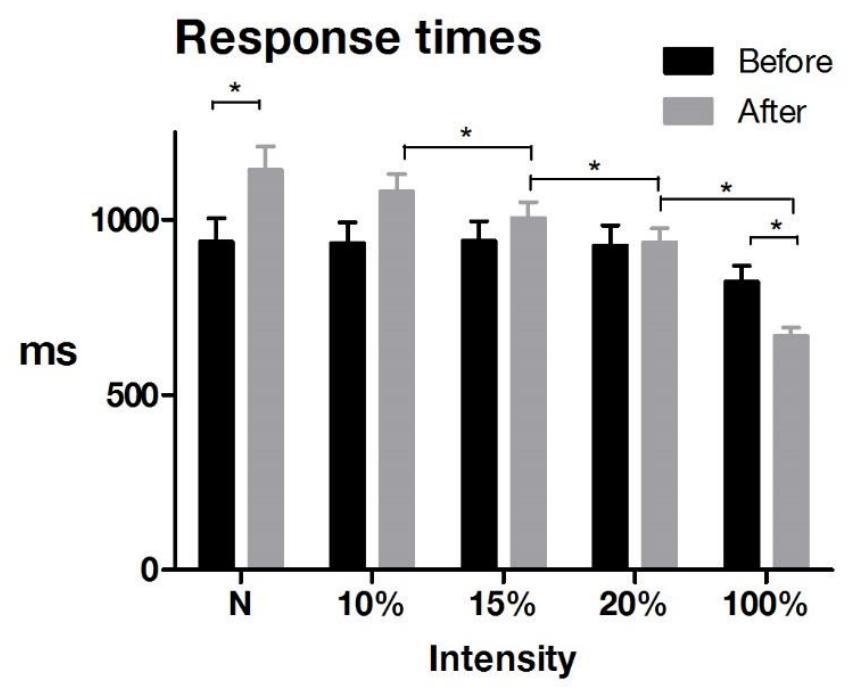

Figure 2: Response times (in milliseconds $(m s)$ ) before and after training for the different expression intensity levels.

Miscategorizations: Proportion error responses were analysed separately for each emotion with the factors Training, Face-set, Intensity and Type (type of miscategorization). I100 was excluded from this analysis due to ceiling performance, particularly after training. Given that 
the effects of Training, Intensity and Face-set are mirrored in the analysis of accuracy, only effects with the factor Type will be reported here. For all three expressions, significant effects were found for Type [for all three Expressions: $\mathrm{F}(2,30) \geq 16.5 ; \mathrm{p}<0.001, \eta_{p}^{2} \geq 0.53$ ], Training $\times$ Type $\left[\mathrm{F}(2,30) \geq 23.2 ; \mathrm{p}<0.001, \eta_{p}{ }^{2} \geq 0.61\right]$ and Intensity $\times$ Type $[\mathrm{F}(4,60) \geq 14.9 ; \mathrm{p} \leq 0.001$, $\left.\eta_{p}{ }^{2} \geq 0.49\right]$ and Training $\times$ Intensity $\times$ Type $\left[\mathrm{F}(4,60) \geq 21.12 ; \mathrm{p}<0.001, \eta_{p}{ }^{2}=0.59\right]$. The threeway interaction was analysed further by comparing the different types of miscategorizations made at pre-and post-test, separately per Intensity and Expression. These analyses showed that compared to the pre-test, significantly fewer happy, sad and surprised expressions were miscategorised as neutral at post-test than at pre-test $\left(p^{\prime} s \leq 0.001\right)$ (see Figure 1). Happy faces were slightly more often miscategorised as surprised at I10 and I15 ( $\left.p^{\prime} s \leq 0.04\right)$, sad faces were more often miscategorised as surprised at I10 and I15 ( $\left.p^{\prime} s \leq 0.007\right)$ whereas miscategorizations of surprised faces for happy or sad expression remained the same. Analysis of the neutral condition also revealed a significant effect of Type $[F(2,30)=64.3$; $\left.\mathrm{p}<0.001, \eta_{p}{ }^{2}=0.81\right]$ which did not interact with other factors: Neutral faces were more often mistaken for sad (37.5\%) than for happy $(7.3 \%)$ or surprised faces $(9.75 \%$, for both comparisons, $p$ 's<0.001).

\subsection{Behavioural measures: Training sessions}

Accuracy: Figure 3 shows training-related changes in accuracy and response times before and after training. Only the lower expression intensity levels were included in analysis of accuracy (10, 15 and 20\%). For the remaining intensity levels, performance for at least one condition was at ceiling (e.g. all participant correctly categorized happy faces with intensity level 25\% in block 2 of session 2). Significant effects were found for Training Session (three sessions) $\left[\mathrm{F}(2,30)=47.9 ; \mathrm{p}<0.001, \eta_{p}{ }^{2}=0.76\right]$, Block (three blocks per training session) $\left[\mathrm{F}(3,45)=7.92 ; \mathrm{p}=0.002, \eta_{p}{ }^{2}=0.34\right]$, Expression $\left[\mathrm{F}(2,30)=12.14 ; \mathrm{p}=0.001, \eta_{p}{ }^{2}=0.45\right]$, Intensity 
$(10,15$ and $20 \%)\left[\mathrm{F}(3,45)=232.3 ; \mathrm{p}<0.001, \eta_{p}{ }^{2}=0.94\right]$, Expression $\times$ Session $[\mathrm{F}(4,60)=4.2$; $\left.\mathrm{p}=0.005, \eta_{p}{ }^{2}=0.22\right]$ and Expression $\times$ Intensity $\left[\mathrm{F}(12,180)=13.34 ; \mathrm{p}<0.001, \eta_{p}{ }^{2}=0.47\right]$. Posthoc analyses of these effects showed that accuracy improved significantly after sessions 1 and $2(p ' s \leq 0.023$ ); within each session performance improved most after block 1 (block 1 vs block 2: $\mathrm{p}=0.02$ ); accuracy was highest for happy and sad compared to surprised faces ( $p$ 's $\leq 0.004)$, and accuracy increased significantly with each intensity level ( $\left.p^{\prime} s<0.001\right)$. Accuracy for happy faces improved after both the first and second training session ( $\left.p^{\prime} s \leq 0.02\right)$, whereas for sad and surprised faces, significant improvements were only found after session 1 ( $\left.p^{\prime} s \leq 0.027\right)$. Furthermore, whilst performance was higher for sad than happy or surprised faces at $10 \%(p ' s \leq 0.015)$, accuracy was higher for both sad and happy faces compared to surprised faces at intensity levels 15 and 20\% ( $p$ 's $\leq 0.03$ ). The analysis of neutral faces only revealed a significant interaction effect of Session Block $[F(4,60)=2.5 ; p=0.047$, $\left.\eta_{p}{ }^{2}=0.16\right]$. Correct responses reduced from block $1(39.8 \%)$ to $2(25.61 \%)$ in session 1 only $\mathrm{p}=0.021)$.

Response times: Response times became faster during training $[\mathrm{F}(2,30)=3.9 ; \mathrm{p}=0.033$, $\eta_{p}{ }^{2}=0.23$ ], significantly so from session 1 to $3(\mathrm{p}=0.015)$. RT also decreased as intensity increased $\left[\mathrm{F}(9,135)=163.7 ; \mathrm{p}<0.001, \eta_{p}{ }^{2}=0.91\right]$ with significant differences between all intensity levels except between 30-35\% ( $\left.p^{\prime} s \leq 0.019\right)$. Responses were faster to happy than to sad or surprised faces $\left[\mathrm{F}(2,30)=13.01 ; \mathrm{p}<0.001 ; \eta_{p}{ }^{2}=0.46\right.$, all $\left.p^{\prime} s \leq 0.01\right]$, although this trend was more pronounced at higher intensity levels $\left[\mathrm{F}(16,240)=4.3 ; \mathrm{p}<0.001, \eta_{p}{ }^{2}=0.22\right]$ : For I20 and higher, responses were faster to happy faces ( $p$ 's $\leq 0.043$ ), whereas at the lowest intensity levels, both happy and sad faces were responded to faster than to surprised faces $(p$ 's<0.045). No significant effects were found in the analysis of the neutral condition. 


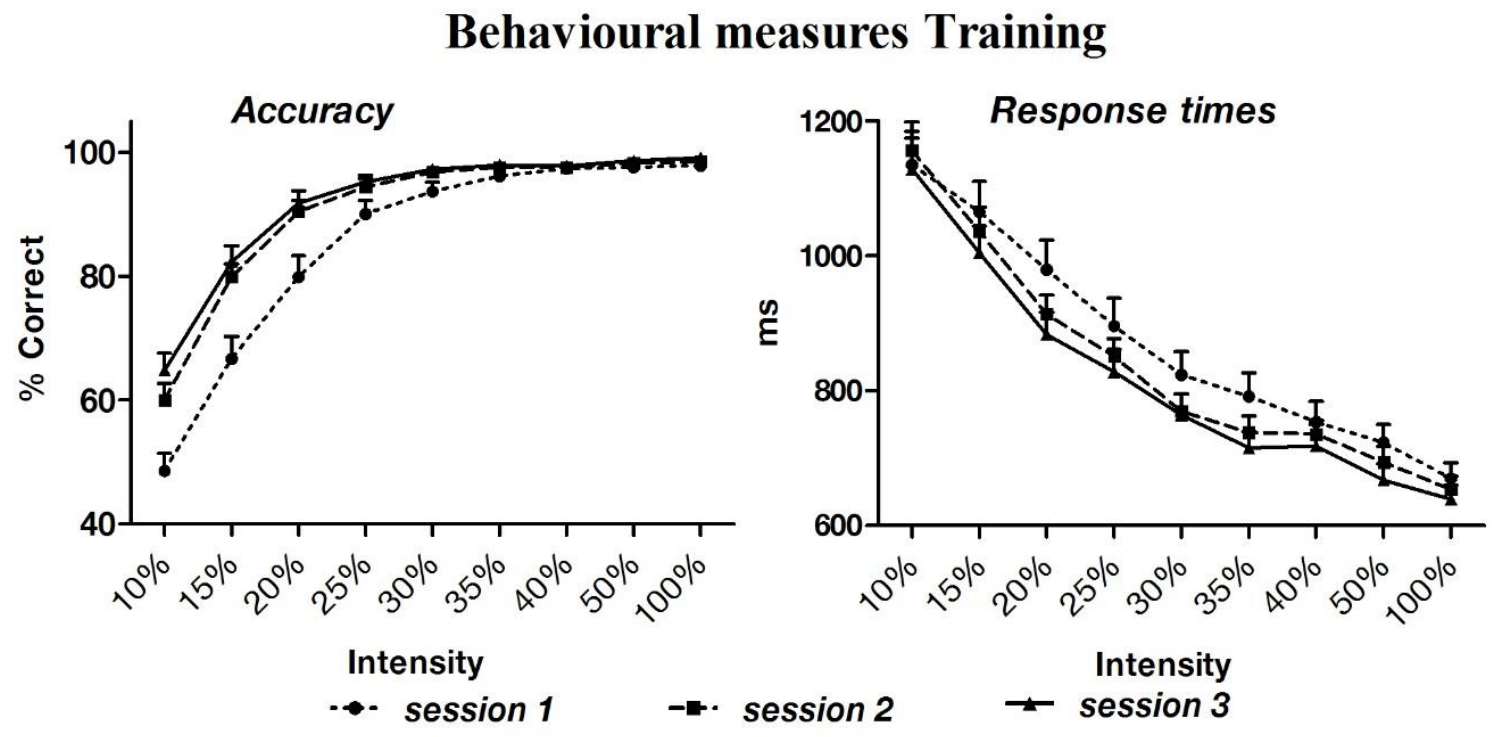

Figure 3: Accuracy (percentage correct) and mean response times (in milliseconds (ms)) for the three training sessions.

Miscategorizations: For analysis of miscategorizations during training, only I10, I15 and I20 were included given ceiling performance in one or more conditions at higher intensities. All faces were more often miscategorised as neutral, but this effect of Type was only significant for sad and surprised faces $\left[\mathrm{F}(2,30) \geq 17.6 ; \mathrm{p}<0.001, \eta_{p}{ }^{2}=0.57\right]$. After neutral, surprised faces were equally often miscategorises as sad or happy (Neutral>Sad/Happy, all $p ' s \leq 0.005$ ), whereas sad faces were significantly more often miscategorised as surprised than as happy (Neutral > Surprised > Happy, all $p{ }^{\prime} \leq 0.034$ ). For both sad and surprised expressions, Type was not significant at I20, resulting in significant Type $\times$ Intensity effects for both expressions $\left[F(4,60) \geq 7.06 ; p<0.001, \eta_{p}{ }^{2} \geq 0.34\right]$. Analysis of the neutral condition revealed a significant effect of Type $\left[\mathrm{F}(2,30)=69.33 ; \mathrm{p}<0.001, \eta_{p}{ }^{2}=0.57\right]$, characterized by a bias towards sad: $\operatorname{Sad}(22.4 \%)>$ surprise $(6.1 \%)>$ happy $\left.(3.4 \%), p{ }^{\prime} \leq 0.0018\right)$. 


\subsection{ERP Measures}

ERP measures were analysed twice: Once including all three expressions but excluding the neutral condition to focus on potential effects of Expression (Analysis 1) and once separately per expression with the neutral condition as an additional level for the factor Intensity (Analysis 2). Analyses focused on modulation of intensity effect within each ERP sessions.

P1: A significant effects of Intensity was found at pre-test only $[\mathrm{F}(3,45)=3.2$; $\left.\mathrm{p}=0.044, \eta_{p}{ }^{2}=0.18\right]$. Before training, the largest difference was between intensity level $10 \%$ and $100 \%$ but differences were not significant when the Bonferroni correction was applied. Inclusion of the neutral condition in Analysis 2 resulted in a non-significant effect of Intensity at pre-test. Intensity effects remained non-significant after training. Analysis of peak latency did not reveal any significant results.

N170: Analysis revealed that N170 amplitude was more pronounced at P8, both before training $\left[\mathrm{F}(1,15)=10.8 ; \mathrm{p}=0.005, \eta_{p}{ }^{2}=0.42\right]$ and after training $[\mathrm{F}(1,15)=5.4, \mathrm{p}=0.034$, $\left.\eta_{p}{ }^{2}=0.27\right]$, but this effect did not interact with other factors. A significantly effect was found for Intensity, both at pre-test $\left[\mathrm{F}(3,45)=16.8 ; \mathrm{p}<0.001, \eta_{p}{ }^{2}=0.53\right]$ and at post-test $\left[\mathrm{F}(3,45)=13.66 ; \mathrm{p}<0.001, \eta_{p}{ }^{2}=0.48\right]$. Amplitude was more negative for 1100 compared to the lower intensity conditions (all $p{ }^{\prime} \leq \leq 0.02$ ). Differences between the lower intensity conditions were not significant. The effect of Intensity was not altered when the neutral condition was included in Analysis 2. N170 to neutral faces was significantly reduced compared to I100 both before and after training ( $p$ 's=0.038) but did not differ from the remaining intensity levels. There were no significant effects in the analysis of the peak latency of N170. 


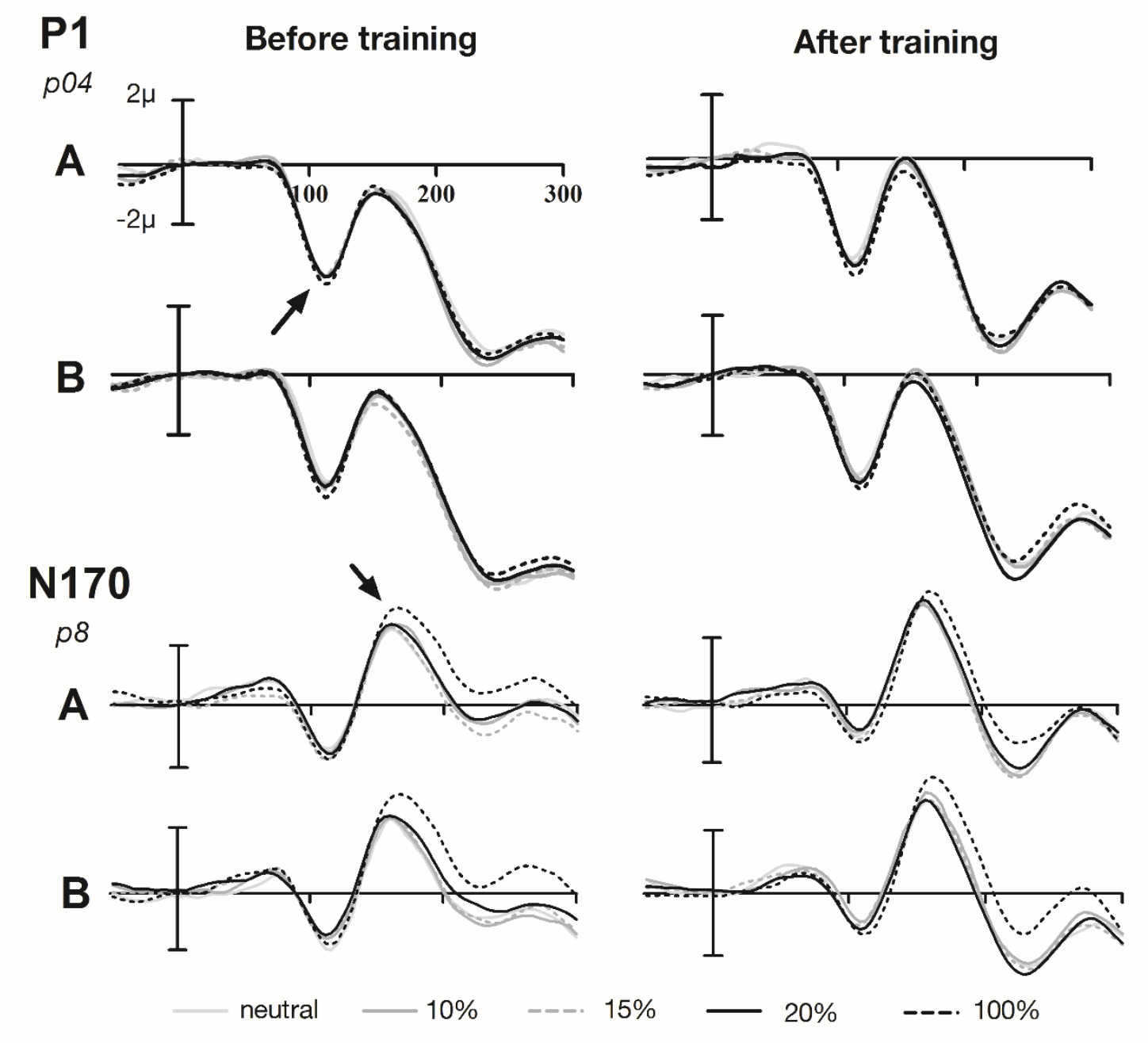

Figure 4: Grand average waveforms: P1 (at p04) and N170 (at p8) for the face set used for training ('A') and new faces (B) for each intensity level (Neutral, 10\%, 15\%, 20\% and 100\%) before and after training

P3/LPP: Analysis 1 and 2 were conducted for each 100ms time-window between 350 and $1550 \mathrm{~ms}$ after stimulus onset. Significant effects of Intensity were only found in the four $100 \mathrm{~ms}$ time-windows between $450 \mathrm{~ms}$ and $850 \mathrm{~ms}$. Figure 5 shows grand-average waveforms at $\mathrm{CP} 2$ before and after training and Table 1 shows the significant post-hoc comparisons of mean amplitude between the different intensity levels for each time-window. 
Before training: Between 450 and 650ms, significant effects were found for Intensity $\left[\mathrm{F}(3,45) \geq 7.92 ; \mathrm{p} \leq 0.002, \eta_{p}{ }^{2} \geq 0.35\right]$ which was characterized by enhanced amplitude for I100 compared to the lower intensity levels $\left(p^{\prime} s \leq 0.006\right)$ and compared to Neutral in Analysis 2 $\left(p^{\prime} s \leq 0.019\right)$. No differences were found between the lower intensity or neutral conditions. The effect of intensity remained significant between 650 and $750[F(3,45)=4.94 ; p=0.018$, $\eta_{p}{ }^{2}=0.24$ ] but at this stage of processing, the effect of Intensity varied with Emotion (between 650 and 850ms: Intensity $\times$ Emotion: $\left.\mathrm{F}(6,90) \geq 3.05 ; \mathrm{p} \leq 0.036, \eta_{p}^{2} \geq 0.17\right]$.

Figure 5 shows that ERP waves associated with happy faces returned to baseline earlier than those associated with less intense facial expressions. Between 650 and 750, this resulted in a significant effect of Intensity for sad and surprised faces (increased amplitude for I100 compared to lower intensities and neutral: $\left.p{ }^{\prime} \leq 0.04\right)$ but not for happy, whereas between 750 and 850, intensity was significant for happy (reduced amplitude for I100 compared to I15 and $\mathrm{I} 20, p$ ' $\leq \leq 0.04$ ) but not for sad and surprised faces. Analysis 1 further revealed a significant Electrode $\times$ Emotion $\times$ Intensity effect between 450 and 550ms $[F(6,90)=3.2 ; p=0.017$, $\left.\eta_{p}{ }^{2}=0.18\right]$. Mean amplitude of I100 faces was enhanced for happy and surprised compared to sad faces at CP2 only ( $\left.p^{\prime} s \leq 0.041\right)$. 


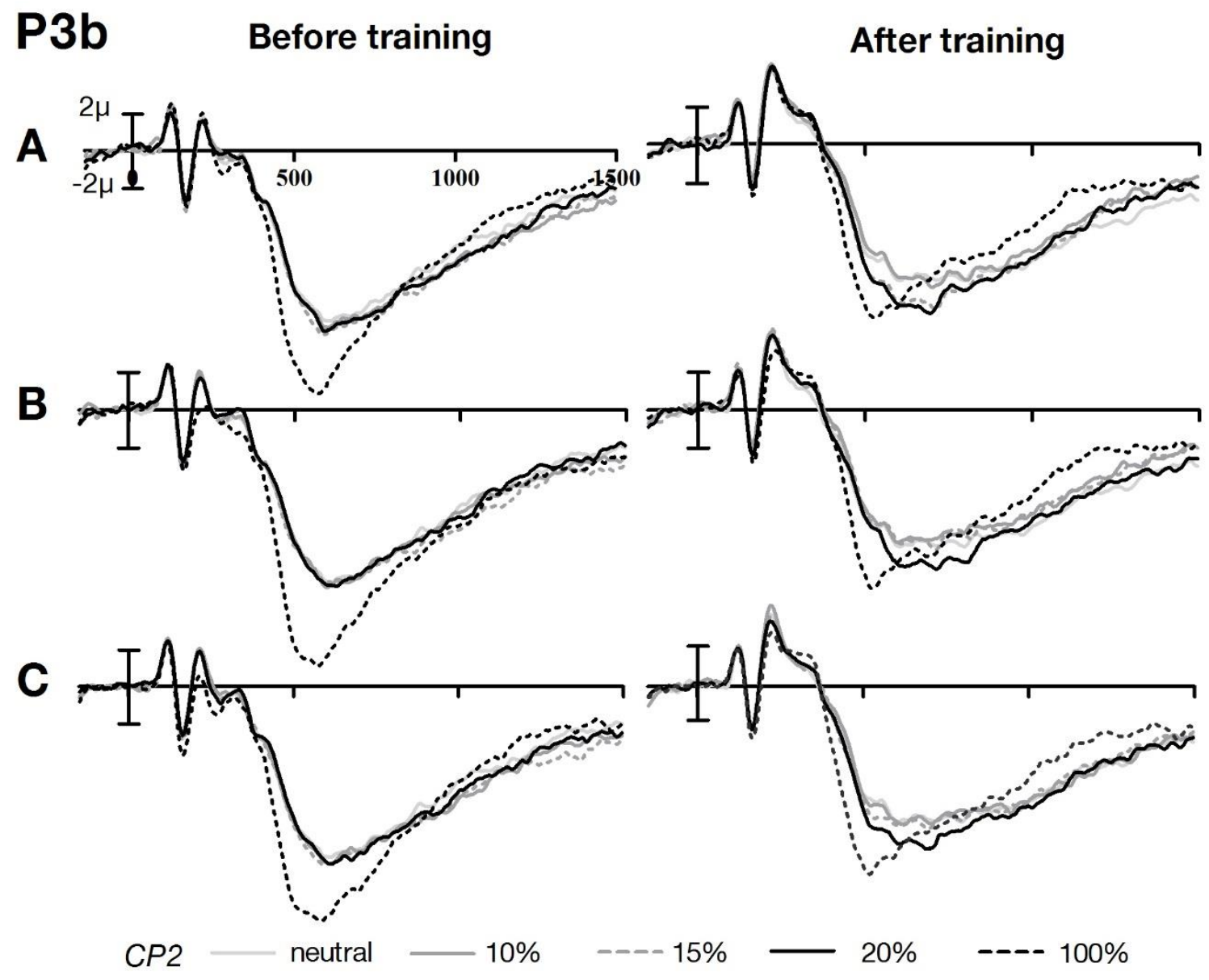

Figure 5: Grand average waveforms: P3b/LPP at CP2 for happy (A), sad (B) and surprised faces (C) for each intensity level (Neutral, 10\%, 15\%, 20\% and 100\%) before and after training.

After training: Figure 5 shows that as predicted, amplitude increased more gradually with facial expression intensity after training. In all four time-windows, significant effects were found for Intensity $\left[F(3,45) \geq 3.67 ; p \leq 0.019, \eta_{p}{ }^{2} \geq 0.19\right]$. A gradual increase in amplitude as a function of expression intensity (here indicated by one or more significant differences of mean amplitude between the lower intensity levels) can be observed between 450 and $650 \mathrm{~ms}$ for happy faces, between 650 and 750ms for sad faces and between 450 and 750ms for surprised faces (see Table 1). 


\begin{tabular}{|c|c|c|c|c|c|}
\hline & & $450-550 \mathrm{~ms}$ & 550-650ms & $650-750 \mathrm{~ms}$ & 750-850ms \\
\hline \multirow[t]{2}{*}{ Happy } & Before & all $\mathrm{LI}<\mathrm{I} 100 \% * * *$ & all $\mathrm{LI}<\mathrm{I} 100 \% * * *$ & $n s$ & all $\mathrm{LI}<\mathrm{I} 100 \%$ * \\
\hline & After & $\begin{array}{l}\text { all } \mathrm{LI}<\mathrm{I} 100 \% * * \\
\mathrm{~N}<\mathrm{I} 10 \% * \\
\mathrm{I} 10 \%<\mathrm{I} 20 \% * \\
\mathrm{I} 10 \%<\mathrm{I} 20 \% *\end{array}$ & $\begin{array}{l}\mathrm{N}<15 \% * * \\
\mathrm{~N}<20 \% * \\
10 \%<15 \% * \\
10 \%<20 \%\end{array}$ & $\begin{array}{l}\mathrm{I} 100 \%<\mathrm{I} 15 \% * \\
\mathrm{I} 100 \%<\mathrm{I} 20 \% *\end{array}$ & I100\%<all LI \\
\hline \multirow[t]{2}{*}{ Sad } & Before & all $\mathrm{LI}<\mathrm{I} 100 \% * * *$ & all $\mathrm{LI}<\mathrm{I} 100 \% *$ & all LI $<\mathrm{I} 100 \% *$ & $\mathrm{~ns}$ \\
\hline & After & all $\mathrm{LI}<\mathrm{I} 100 \% * * *$ & $n s$ & $\begin{array}{l}\mathrm{N}<\mathrm{I} 20 \% * * \\
\mathrm{I} 10 \%<\mathrm{I} 20 \% * * \\
\mathrm{I} 15 \%<\mathrm{I} 20 *\end{array}$ & ns \\
\hline \multirow[t]{2}{*}{ Surprised } & Before & all $\mathrm{LI}<\mathrm{I} 100 \% * * *$ & all $\mathrm{LI}<\mathrm{I} 100 \% * * *$ & all LI $<\mathrm{I} 100 \% *$ & ns \\
\hline & After & $\begin{array}{l}\text { all } \mathrm{LI}<\mathrm{I} 100 \% * * * \\
\mathrm{~N}<\mathrm{I} 20 \% *\end{array}$ & $\begin{array}{l}\mathrm{I} 10 \%<\mathrm{I} 20 \% * * \\
\mathrm{I} 15 \%<\mathrm{I} 20 \% * *\end{array}$ & $\begin{array}{l}\mathrm{N}<\mathrm{I} 20 \% * \\
\mathrm{I} 10 \%<\mathrm{I} 20 \% * * \\
\mathrm{I} 15 \%<\mathrm{I} 20 \% *\end{array}$ & ns \\
\hline
\end{tabular}

Table 1: Results of post-hoc comparisons of Intensity on mean P3b/LPP mean amplitude before and after training. 'All LI' = All lower intensities (neutral, 10\%, 15\% and 20\%), 'N' $=$ Neutral, 'I10' = Intensity level 10\%, 'I15' = intensity level 15\%, 'I20' = Intensity level $20 \%$, 'I100' = Intensity level 100\%. With Bonferroni correction: $*=p \leq 0.05, * *=p \leq 0.01, * * *=$ $p<0.001$

ERP waves associated with happy faces returned to baseline faster than sad or surprised faces after training, reflected in reduced amplitude for I100 compared to lower intensity expressions between 650 and 850ms (see Table 1). Analysis 1 further revealed a significant effect of Emotion between 450 and $650 \mathrm{~ms}\left[\mathrm{~F}(2,30) \geq 6.7 ; \mathrm{p} \leq 0.008, \eta_{p}{ }^{2} \geq 0.31\right]$, due to enhanced amplitude for happy and surprised compared to sad faces ( $p$ 's $\leq 0.05)$, and a three-way 
interaction effect of Electrode $\times$ Emotion $\times$ Intensity $\left[F(6,90) \geq 3.26 ; p \leq 0.006, \eta_{p}{ }^{2} \geq 0.18\right]$. At CP1, mean amplitude for happy and surprised faces was enhanced compared to sad faces at I15 and I20 between 450 and 550ms $\left(p^{\prime} s \leq 0.014\right)$ and for all four intensity levels between 550 and $650 \mathrm{~ms}(p$ 's $\leq 0.036)$. The effect of emotion was more subtle at CP2: Between 450 and 650 , mean amplitude for happy faces was greater than for sad or surprised faces at I20 ( $p^{\prime} s \leq 0.016$ ), whereas between 550 and $650 \mathrm{~ms}$, amplitude for happy faces was also enhanced compared to sad faces at I15 $(\mathrm{p}=0.02)$ and for surprised faces compared to sad faces at I20 $(\mathrm{p}=0.008)$.

Significant effects of Intensity were also found in P3 peak-latency at pre-test $\left[\mathrm{F}(3,45)=23.7 ; \mathrm{p}<0.001, \eta_{p}{ }^{2}=0.65\right]$ and post-test $\left[\mathrm{F}(3,45)=34.7 ; \mathrm{p}<0.001, \eta_{p}{ }^{2}=0.72\right]$. In all analysis, P3 peaked significantly faster for I100 compared to all lower intensity levels (including neutral) $\left(p^{\prime} s \leq 0.001\right)$.

\subsection{Additional analyses}

Whilst the predictions for this study focused on the effect of expression intensity before and after training, Figure 5 shows that P3b/LPP substantially reduced after training. To analyse whether this was a general trend for all components or restricted to P3b/LPP, mean amplitude was compared before and after training for $100 \%$ intense and low-intense facial expressions (including neutral). This analysis revealed a significant reduction for P3b/LPP amplitude for expressions with $100 \%$ intensity only [t(15) $=2.87 ; \mathrm{p}=0.012]$. All remaining comparisons were not significant.

Correlations: Peak latency of P3/LPP and mean response times (collapsed for trained and new faces and electrode) correlated significantly after training $(r=0.23, p=0.039)$. This relationship was not significant at pre-test. A similar trend was found for the relationship 
between accuracy and P3b/LPP mean amplitude (collapsed for trained and new faces, electrode and time-window): A significant positive linear relationship was only found after training $(\mathrm{r}=0.36, \mathrm{p}=0.001)$.

\section{DISCUSSION}

The present study revealed that behaviourally, response feedback-based training with subtle facial expressions significantly improved categorization accuracy. This effect was similar for all three expressions and was observed for the face-set used during training and for new faces, suggesting strong transfer-effects of learning. ERP data showed that these behavioural improvements were not associated with emotion-related ERP modulations at early stages of facial expression processing. Whilst training increased correct categorization substantially at lower intensity levels, emotion-related P1 and N170 modulations were largely unaffected by this behavioural improvement. The earliest training-related changes were observed from $450 \mathrm{~ms}$ onwards, characterized most noticeably by a more gradual increase in amplitude for low intensity expressions after training. These findings suggest that feedbackbased training in categorization of subtle facial expressions predominantly influenced postperceptual processes.

At earlier stages of processing (N170), only the $100 \%$ intense expressions elicited a reliable emotion-related modulation compared to neutral faces, whereas ERPs to lowintensity expressions were indistinguishable from neutral and from each other, both before and after training. The absence of a more gradual intensity effect for N170 amplitude after training, despite high accuracy for all low intensity levels, may suggest that successful discrimination between emotional and neutral faces (Luo et al, 2010) can only be achieved for more intense facial expressions at this early stage whereas the fine-grained 
discriminations between low intensity expressions and neutral may rely on more elaborate processes that extend into later stages. Alternatively, the ERP methodology may not be sensitive enough to reveal modulations in N170 amplitude when the differences between intensity levels are small and the levels that are compared are all low. Only few studies have investigated N170 effects of facial expression intensity levels directly. Sprengelmeyer et al (2006) reported significant enhancements of N170 amplitude with increasing expression intensity but used larger intensity differences (50\%, 100\%, 150\%). Utama et al (2009) used low intensity levels and smaller differences $(15,30,40,75$ and 100\%) and found that N170 amplitude correlated positively with intensity ratings, although amplitude differences between the lower intensity levels were not analysed statistically. Moreover, in Utama et al (2009) each emotional face was preceded by a neutral face, which may have influenced the ease of discrimination of emotional from neutral faces at earlier stages of processing. To which extent expression intensity modulation of N170 is specific to a particular methodology will require further investigations. Our findings suggest that in the present study, N170 amplitude did not discriminate between low intensity expressions even when the majority of these expressions were categorized correctly after training.

Between 450 and $850 \mathrm{~ms}$ after stimulus onset, amplitude elicited by subtle facial expressions enhanced more gradually with increasing intensity level after training. This effect was absent before training and coincided with a significant increase of correct responses (as labelled during training) and reduced categorizations of ambiguous expressions as neutral. The latency of this effect is consistent with the time-windows previously associated with P3b and LPP modulations by facial expressions (Eimer and Holmes, 2002; Hajcak et al, 2010; Duval et al, 2013). In accordance with the assumption that emotional content attracts more attentional processing resources (Lang and Bradley, 2010), enhancing P3b/LPP (Hajcak et al, 2010; Duval et al, 2013), the change in categorization of low-intensity expressions from 
neutral to the labelled emotion after training seems to have increased the motivational salience for a proportion of these faces, most noticeably faces with $20 \%$ intensity level expressions. This effect was more pronounced for happy faces: Amplitude elicited by low intensity happy expressions increased significantly for intensity levels $15 \%$ and $20 \%$ compared to lower intensities, whereas for sad and surprised faces, enhancements were only observed for the $20 \%$ intensity level. The enhanced P3b/LPP effect for happy faces did not coincide with more pronounced increments in performance measures: Training-related behavioural improvements were statistically similar for all three facial expressions and overall accuracy was comparable for happy and sad expressions. One possible explanation for this trend could be that ceiling performance was reached after training. Accuracy for low intensity happy and sad faces was very high after training ( $80 \%)$ and no additional performance improvements were achieved after the first training session. A plateau in training effects after the first training session for low intensity expressions (20\%) has been found in our previous study (Pollux et al, 2014). Alternatively, the performance measures used in the present study may not have been sufficiently sensitive to reveal small changes in recognition of subtle expressions. More expression intensity levels (e.g. in steps of $2 \%$ ) could be used in future training studies to explore this question further.

Behavioural improvements were similar for trained and new faces, consistent with our previous findings (Pollux et al, 2014) and training-related ERP modulations were indistinguishable for both face-sets, suggesting strong transfer-effects of learning. The exact nature of the processes underlying learning in the present study can only be speculated. It is assumed that changes in categorization of subtle facial expressions previously perceived as neutral required more elaborate, time-demanding analysis of facial information that extends into later stages of processing. This idea seems consistent with the observation that response times for the lowest intensity expressions significantly increased after training. In line with 
the construction hypothesis for emotion perception (Gendron et al, 2012), which highlights the constructive (instead of passive) processes underlying emotion perception and the importance of language in these processes, extended analysis may involve more elaborate matching of diagnostic facial information in subtle facial expressions with representations of intense expressions typically associated with the emotion labels (Gendron et al, 2012; Lindquist and Gendron, 2013). Feedback during training may have resulted in engagement of this fine-grained analysis when confronted with any subtle facial expression after training, which could explain the strong transfer effects to new faces for both behavioural and ERP measures.

Training reduced overall P3b/LPP amplitude significantly for the $100 \%$ intense facial expressions whereas this effect was absent for lower intensity levels and for components associated with earlier stages of processing (N170 and P1). A possible explanation for this effect may be related to the influence of stimulus repetition on LPP amplitude. Codispote et al (2006) previously found that repeated presentation of positive or negative images reduces overall magnitude of LPP, which was attributed to habituation and a moderation of attentional resource allocation. However, positive and negative pictures still elicited stronger LPP responses than neutral images, suggesting that the affective modulation remained the same. A similar effect of habituation may underlie the reduction in LPP amplitude after training for the most intense facial expressions.

One methodological issue that requires consideration is the possibility that the perceived probability of 'neutral' being the correct response changed after training and may have resulted in a general shift away from selecting the neutral response option, particularly for the low intense expressions. On its own, this potential response bias could not easily account for the training-related behavioural and ERP effects observed in the present study. A change in response bias would imply that selection of other response options was random, 
without actual improvement in recognition of subtle facial expression. Given the significant correlation found between accuracy and P3b/LPP amplitude after training, suggesting that accuracy was related to the motivational salience of emotional faces (Oloffson et al, 2008; Hajcek and MacNamara, 2010; Duval et al, 2013), it is highly unlikely that increased accuracy was obtained without actual improvement in recognition of facial expressions. At present, it is uncertain to which extent a potential response bias may have influenced the behavioural effects of training and will require further investigation, possibly by manipulating the proportion of neutral faces during the training sessions.

A few additional methodological issues may require consideration in future studies. Firstly, participants were predominantly female in this study. Women have previously been reported to perform better in facial expression categorization tasks compared to men (Vassalo et al, 2009). An equal balance between male and female participants would have allowed analysis of potential differences in achievable benefits of training for men and women. Secondly, mental state of participants was not recorded. Given that facial expression categorization skills are impaired in people with social anxiety (Heuer et al, 2010) and major depression (Joormann and Gotlieb, 2006), it may be important to investigate whether subclinical levels of depression or anxiety in a typical population could affect expression recognition skills or the potential benefit of facial expression training.

An outstanding question is whether behavioural improvements observed in the present study would transfer to contexts beyond the laboratory. Successful reduction of anger biases using feedback based re-labelling has previously been associated with remarkable changes in subjective feelings of anger and in social interaction (Penton-Voak et al, 2013), clearly indicating that changes in categorization of facial expressions transfers to new situations. It will be interesting to learn in future studies whether improved sensitivity to recognizing 
emotions in subtle facial expressions demonstrated here in adults, and previously in children (Pollux et al, 2014), could be associated with similar benefits for social interaction.

The neural structures involved in the enhanced motivational salience of subtle facial expressions after training are likely to be wide-spread given the number of structures suggested to be involved in generation of LPP (Sabetinelli et al, 2007; Liu et al, 2012). Using a LPP-BOLD coupling procedure, where single trial LPP estimates were correlated with single trial BOLD responses, Liu et al (2012) identified generators in the occipito-temporal, temporal and parietal regions in addition to deeper structures such as insula and amygdala, the latter group supporting involvement of the motivational system in allocation of attentional resources to emotional information. It was further argued that processing in visual cortices may be enhanced by subcortical structures and frontal cortices (structures that are involved emotion processing) in a re-entrant fashion (Pressoa and Adolphs, 2010). Future studies could use the LPP-BOLD coupling methodology to investigate if as assumed, improved emotion perception combined with enhanced P3b/LPP amplitude for subtle facial expressions after training is associated with activation in subcortical structures such as insula and amygdala.

To conclude, the likelihood that subtle facial expressions, previously perceived as neutral, are categorized correctly, increases after feedback-based training. This improvement coincides with modulations of P3b/LPP amplitude, suggesting enhanced motivational salience of subtle facial expressions after training. ERP components associated with earlier stages of processing were unaffected, indicating that training influenced mostly postperceptual processing stages. Effects of training were comparable for trained faces and face images not used during training, suggesting strong transfer effects. Future studies are needed to investigate to which extent training effects transfer to contexts beyond the laboratory. 
Acknowledgements: Acknowledgment to Sonia Montemurro and Connor Wyatt for their support in data collection and analysis for this project.

\section{REFERENCES}

Adolphs R, Gosselin F, Buchanan TW, Tranel D, Schyns PG, \& Damasio AR (2005), A mechanism for impaired fear recognition after amygdale damage. Nature 433:68-72.

Baron-Cohen S (1995). Mindbliness: An essay on autism and theory of mind. Boston: MIT Press/Bradford books.

Batty M, Taylor MJ (2003), Early processing of the six basic facial emotional expression. Cogn Brain Res 17: 613-620.

Benton S, Deouell LY (2000), Structural encoding and identification in face processing: ERP evidence for separate mechanisms. Cogn Neurophys 17:35-54.

Campanella S, Quinet P, Bruyer R, Crommelinck M, Guerit JM (2002), Categorical perception of happiness and fear facial expressions: An ERP study. J Cogn Neurosci 14:210227.

Carton JS, Kessler EA, Pape CL (1999), Nonverbal decoding skills and relationship wellbeing in adults. $\mathrm{J}$ Nonverb Beh 23:91-100. 
Codispoti M, Ferrari V, Bradley M (2006). Repetitive picture processing: Autonomic and cortical correlates. Brain Res, 1068:213-220.

Combs DR, Chapman D, Waguspack J, Basso MR, Penn DL (2011), Attention shaping as a means to improve emotion perception deficits in outpatients with schizophrenia and impaired controls. Schiz Res, 127:151-156.

Cuthbert BN, Schupp HT, Bradley MM, Birbaumer N, Lang PJ (2000), Brain potentials in affective picture processing: Covariation with autonomic arousal and affective report. Biol Psy, 52:95-111.

Da Silva Ferreira GC, Crippa J-S, de Lima Osório F (2014), Facial emotion processing and recognition among maltreated children: a systematic literature review. Front Psy, 5, 1-10.

Duval ER, Moser JS, Huppert JD, Simons RF (2013), What's in a face? The late positive potential reflects the level of facial affect expression. J Psychophys, 27:27-38.

Edwards J, JacksonH, Pattison PE (2002), Emotion recognition via facial expression and affective prosody in schizophrenia: A methodological review. Clin Psy Rev, 22:789-832.

Eger E, Jednak A, Iwaki T Skrandies W (2003), Rapid extraction of emotional expression: evidence from evoked potential fields during brief presentation of face stimuli. Neuropsychologia 4:808-817. 
Eimer M, Holmes A (2002). An ERP study on the time course of emotional face processing. NeuroReport 13:1-5.

Eimer M, Holmes A, McGlone F (2003). The role of spatial attention in the processing of facial expression: An ERP study of rapid brain responses to six basic emotions. Cogn Affect Beh Neurosc 3:97-110.

Gendron M, Lindquist KA, Barsalou L, Barrett LF (2012), Emotion words shape emotion percepts. Emotion 12:314-325.

Golan O, Ashwin E, Granader Y, McClintock S, Day K, Leggett V, Baron-Cohen S (2010), Enhancing emotion recognition in children with autism spectrum conditions: An intervention using animated vehicles with real emotional faces. J Autism Dev Disorders 40:269-279.

Guo K (2012), Holistic gaze strategy to categorize facial expression of varying intensities. PLOSone 7:e42585.

Hajcak G, MacNamara A (2010), Event-related potentials, emotion and emotion regulation: An integrative review. Dev Neuropsy 35:129-155.

Heuer K, Lange W, Isaac L Rinck M, Becker E (2010), Morphed emotional faces: Emotion detection and misinterpretation in social anxiety. J Beh Therapy Exp Psych 41:418-425.

Hinojosa JA, Mercado F, Carretié L (2015), N170 sensitivity to facial expression: A metaanalysis. Neurosc Biobehl Rev 55:498-509. 
Itier R J, Taylor M J (2004), N170 or N1? Spatiotemporal differences between object and face processing using ERPs. Cerebral Cortex 14:132-142.

Joormann J, Gotlib I (2006), Is this happiness I see? Biases in the identification of emotional facial expressions in depression and social phobia. J Abnormal Psy 115:705-714.

Lang PJ, Bradley MM (2010), Emotion and the motivational brain. Biol Psy, 84:437-450.

Lindquist KA, Gendron M, (2013). What's in a word? Language constructs emotion perception. Emotion Rev 5:66-71.

Liu Y, Huang J, McGinnis-Deweese M, Keil A, Ding M (2012), Neural substrate of the late positive potential in emotional processing. The J Neurosci 32, 14563-14572.

Luckhaus C, Fromman N, Stroth S, Brinkmeyer J, Wölwer W (2013), Training of affect recognition in Schizophrenia patients with violent offenses: Behavioral treatment effects and electrophysiological correlates. Soc Neurosci 8:503-514.

Lundqvist D, Flykt A, Ohman A (1998), The Karolinska Directed Emotional Faces - KDEF, CD ROM from Department of Clinical Neuroscience, Psychology section, Karolinska Institute, ISBN 91-630-7164-9.

Luo W, Feng W, He W, Wang N, Luo Y (2010). Three stages of facial expression processing: ERP study with rapid serial visual presentation. NeuroImage 49:1857-1867. 
Münte TF, Brack M, Grooheer O, Wieringa BM, Matzke M, Johannes S (1998). Brain potentials reveal the timing of face identity and expression judgements. Neurosci Res 30:2430.

Norwicki S Jr, Duke MP (1994). Individual differences in the nonverbal communication of affect: The diagnostic analysis of nonverbal accuracy scale. J Nonverb Beh, 19, 9-35.

Olofsson JK, Nordin S, Sequeira H, Polich J (2008), Affective picture processing: An integrative review of ERP findings. Biol Psych 77:247-265.

Penn DL, Addington J, Pinkham A, (2006), Social cognitive impairments. In: Lieberman, J. A., Stroup, T. S., Perkins, D.O. (Eds.). American Psychiatric Association Textbook of Schizophrenia. American Psychiatric Publishing Press, Inc., Arlington, VA, pp. 261-274.

Penton-Voak IS, Thomas J, Gage SH, Murran M, McDonald S, Manufò (2013), Increasing recognition of happiness in ambiguous facial expressions reduces anger and aggressive behaviour. Psy Sci 24:688-697.

Pollak SD, Messner M, Kisler DJ, Cohn JF (2009), Development of perceptual expertise in emotion recognition. Cognition 110:242-247.

Pollux P-J, Hall S, Guo K (2014), Facial expression training optimises viewing strategy in children and adults. PLOSone 9:e105418. 
Pourtois G, Grandjean D, Sander D, Vuilleumier P (2004), Electrophysiological correlates of rapid spatial orienting towards fearful faces. Cerebral Cortex 14:619-633.

Pressoa L, Adolphs R (2010). Emotion processing and the amygdala: From a 'low road' to 'many roads' of evaluating biological significance. Nature Neurosci Rev 11:773-782.

Roisson B, Campanella S, Gomez CM, Delinte A, Debatisse D, Liard L, Dubois S, Bruyer, R, Crommelinck M, Guerit JM (1999), Task modulation of brain activity related to familiar and unfamiliar face processing: An ERP study. Clin Neurophys 110:449-462.

Sabatinelli D, Lang P J, Keil A, Bradley MM (2007), Emotional perception: Correlation of functional MRI and event-related potentials. Cerebral Cortex 17:1085-1091.

Sachs G, Winklbaur B, Jagsch R, Lasser I, Ksyspin-Exner I, Frommann N, Wölwer W (2012), Training of affect recognition (TAR) in schizophrenia-Impact on functional outcome. Schiz Res 138:262-267.

Sadeh B, Polipsky I, Zhdanov A, Yovel G (2010), Event-related potential and functional MRI measures of face-selectivity are highly correlated: a simultaneous ERP-fMRI investigation. Human Brain Mapping 31:140-1501.

Schupp HT, Cuthbert BN, Bradley MM, Cacciaoppo JT, Ito T, Lang PJ (2000), Affective picture processing: The late positive potential is modulated by motivational relevance. Psychophysiology 37:257-261. 
Sprengelmeyer R, Jentzsch I (2006), Event related potentials and the perception of intensity in facial expressions. Neuropsychologia 44:2899-2906.

Sullivan S, Ruffman T, Hutton SB (2007), Age differences in emotion recognition skills and the visual scanning of emotion faces. J Gerontol: Psy Sci 62:53-60.

Tamamiya Y, Hiraki K (2013), Individual differences in the recognition of facial expressions: An event-related potentials study. PLOSone 8:e57325.

Utama NP, Takemoto A, Koike Y, Nakamura K. (2009), Phased processing of facial emotion: An ERP study. Neurosci Res 64:30-40.

Vassalo S, Cooper SL, Douglas JM (2009). Visual scanning in the recognition of facial affect: Is there an observer sex difference? J Vision 9:1-10.

Vuilleumier P, Pourtois G (2007). Distributed and interactive brain mechanisms during emotion face perceptions: evidence from functional neuroimaging. Neuropsychologia 45:174-194.

Werheid K, Gruno M, Kathmann N, Fischer H, Almkvist O, Winblad B (2010). Biased recognition of positive faces in aging and amestic mild cognitive impairment. Psy Aging $25: 1-15$.

Williams LM, Palmer D, Liddell BJ, Song L, Gordon E (2006), The when and where of perceiving signals of threat versus non-threat. NeuroImage 31:458-467. 
Zhang D, Luo W, Luo Y (2013), Single-trial ERP analysis reveals facial expression category in three-stage scheme. Brain Res 1512:78-88.

\section{CAPTURES FOR FIGURES AND TABLE}

Figure 1: Left column: Percentage correct responses (\% correct) for faces-set used for training ('T-faces') and faces not used for training ('N faces') by Intensity and Expression ( $\mathrm{A}=$ Happy, $\mathrm{B}=\mathrm{Sad}, \mathrm{C}=$ Surprised). Right column: Percentage miscategorizations for low expression intensity only $(10 \%, 15 \%$ and $20 \%)$, collapsed over intensity level and face-set, before and after training (H=Happy, $\mathrm{S}=\mathrm{Sad}, \mathrm{Su}=\mathrm{Surprised}, \mathrm{N}=$ Neutral.

Figure 2: Response times (in milliseconds (ms)) before and after training for the different expression intensity levels.

Figure 3: Accuracy (percentage correct) and mean response times (in milliseconds (ms)) for the three training sessions.

Figure 4: Grand average waveforms: P1 (at p04) and N170 (at p8) for the face set used for training ('A') and new faces (B) for each intensity level (Neutral, 10\%, 15\%, 20\% and 100\%) before and after training 
Figure 5: Grand average waveforms: P3b/LPP at CP2 for happy (A), sad (B) and surprised faces (C) for each intensity level (Neutral, 10\%, 15\%, 20\% and 100\%) before and after training.

Table 1: Results of post-hoc comparisons of Intensity on mean P3b/LPP mean amplitude before and after training. 'All LI' = All lower intensities (neutral, 10\%, 15\% and 20\%), 'N'= Neutral, 'I10' = Intensity level 10\%, 'I15' = intensity level 15\%, 'I20' = Intensity level 20\%, $' \mathrm{I} 100 ’=$ Intensity level 100\%. 